\title{
"Metronomic" chemotherapy in advanced soft tissue sarcomas
}

\author{
Antoine Italiano $\cdot$ Maud Toulmonde $\cdot$ Barbara Lortal \\ Eberhard Stoeckle • Delphine Garbay · Guy Kantor • \\ Michèle Kind · Jean-Michel Coindre · Binh Bui
}

Received: 5 December 2009/Accepted: 3 February 2010/Published online: 25 February 2010

(C) Springer-Verlag 2010

\begin{abstract}
Purpose Angiogenesis plays a crucial role in metastatic progression of soft tissue sarcomas (STS). Endothelial cells are the primary target of metronomic chemotherapy. We report the safety and the efficacy of metronomic chemotherapy in metastatic STS patients.

Methods The medical charts of 26 metastatic STS patients treated at Institut Bergonie (Bordeaux, France) with metronomic etoposide $(100 \mathrm{mg} / \mathrm{day}$ orally for 21 consecutive days, repeated every 4 weeks) were reviewed by two independent investigators.

Results Median age was 49. All but three patients received prior treatment with doxorubicin and/or ifosfamide. One patient (4\%) had partial response and 11 patients (42\%) had stable disease for more than 24 weeks.
\end{abstract}

A. Italiano and M. Toulmonde have equally contributed to the study.

A. Italiano $(\varangle) \cdot$ M. Toulmonde $\cdot$ D. Garbay $\cdot$ B. Bui

Department of Medical Oncology, Institut Bergonié,

229 cours de l'Argonne, 33076 Bordeaux Cedex, France

e-mail: italiano@bergonie.org

\section{B. Lortal}

Department of Pharmacy, Institut Bergonié, Bordeaux, France

E. Stoeckle

Department of Surgery, Institut Bergonié, Bordeaux, France

G. Kantor

Department of Radiotherapy,

Institut Bergonié, Bordeaux, France

M. Kind

Department of Radiology, Institut Bergonié, Bordeaux, France

J.-M. Coindre

Department of Pathology, Institut Bergonié, Bordeaux, France
The 6-month and the 1-year progression-free survival rates were $42 \%$ [95\% CI: 23; 61] and 23\% [95\% CI: 7; 39], respectively. The 6-month and the 1-year overall survival rates were $69 \%$ [95\% CI: 51; 87] and 31\% [95\% CI: 13 ; 49], respectively. Two patients experienced grade 4 febrile neutropenia and one of them died of sepsis.

Conclusion In this series, metronomic etoposide was associated with significant clinical activity in STS. Further prospective investigations are necessary to identify those patients who are more likely to benefit from this strategy.

Keywords Metronomic - Chemotherapy · Sarcoma . Angiogenesis

\section{Introduction}

The prognosis of patients who develop metastatic soft tissue sarcoma (STS) remains poor. Despite the best standard chemotherapy available (anthracyclines and ifosfamide), the median survival is only 12 months and long-term survival is observed in less than $10 \%$ of patients [1]. Therefore, new effective systemic treatments are urgently needed for this patient population.

There are several lines of evidence indicating that sarcomas are angiogenesis dependent. Sarcomas are known to express potent angiogenic factors [2, 3], which may play a crucial role in the formation of metastasis. For instance, studies in rhabdomyosarcoma have shown that higher levels of VEGF and MMP expression may contribute to a metastatic phenotype $[4,5]$. Therefore, tumor angiogenesis could be a crucial therapeutic target in soft tissue sarcoma patients.

Metronomic chemotherapy refers to the frequent, even daily, administration of cytotoxic agents at doses 
significantly less than the maximum-tolerated dose, with no prolonged rest periods [6]. Unlike traditional chemotherapy schedules, the main targets of which are presumed to be proliferating tumor cells, the main targets of metronomic chemotherapy are the endothelial cells of the growing vasculature of a tumor [7]. This "anti-angiogenic" effect, demonstrated in several preclinical models, result from the continuous exposure of the proliferating tumor endothelial cells to the cytotoxic drug, thereby limiting their opportunity to repair DNA damage. The therapeutic role of metronomic chemotherapy in soft tissue sarcomas has been suggested in preclinical studies demonstrating the efficacy and the safety of low-dose cyclophosphamide in rat and dog sarcoma models $[8,9]$.

We report here a mono-institutional experience that tested the activity of a metronomic chemotherapy in metastatic soft tissue sarcoma patients.

\section{Patients and methods}

\section{Patients}

From 1995 to 2008,746 patients all $\geq 15$ years with a soft tissue sarcoma were admitted at Institut Bergonie (Bordeaux, France) for the management of a first tumor event and were included in the database of the French Sarcoma Group (GSF). For all patients, a histological review was performed by the members of the GSF pathological subcommittee. The histological diagnosis was established according to the World Health Organization Classification of Tumors [10]. The histological grade was determined after central review as previously described according to the Fédération Nationale des Centres de Lutte Contre le Cancer (FNCLCC) grading system [11]. One hundred and sixty-nine patients (23\%) presented with metastatic disease during their clinical course. Among them, twenty-six patients received metronomic chemotherapy during the course of their disease. Their medical charts were reviewed by two independent investigators who were not involved in the care of the patients. This study was approved by the local ethics committee.

\section{Treatment}

Metronomic chemotherapy consisted of etoposide $100 \mathrm{mg} /$ day (fixed dose) orally for 21 consecutive days, repeated every 4 weeks. A blood cell count was performed every week. If neutrophils or platelets were below $1000 / \mathrm{mm}^{3}$ or $100,000 / \mathrm{mm}^{3}$, respectively, treatment was delayed until recovery for a maximum of 2 weeks. If no recovery took place, a dose reduction was made from 100 to 75 or $50 \mathrm{mg}$. Treatment dose was also reduced in case of non- hematological grade 3 or 4 toxicity or persistent grade 2 toxicity despite adequate symptomatic treatment. The treatment was continued until patients experienced disease progression, unacceptable toxicity, or death.

\section{Treatment evaluations}

The best response to treatment was evaluated according to Response Evaluation Criteria in Solid Tumors [12]. Progression-free survival (PFS) was defined as the time from the start of metronomic chemotherapy until disease progression, death or last patient contact. Overall survival (OS) was defined as the time from the start of metronomic chemotherapy until death or last patient contact. National Cancer Institute Common toxicity Criteria (version 3.0) were used to classify adverse events.

Statistical analysis

The statistical analysis of baseline demographics and clinical outcome is based on all data available up to the cutoff date of June 30, 2009. Survival rates were estimated with the use of the Kaplan-Meier method. Descriptive statistics were used to show the distribution of variables in the population. Differences between groups were evaluated by the Chi square test or Fisher's exact test for categorical variables and $t$ test for continuous variables. Prognostic factors were planned to be identified by univariate and multivariate analyses by using Cox regression model. Variables associated with PFS and/or OS with a $P$ value $<0.05$ in the univariate analysis were planned to be included in the multivariate regression. Analyses were performed using SPSS 12.0 statistical software (IPSS Inc., Chicago, USA). All statistical tests were two sided, and $P<0.05$ indicated statistical significance.

\section{Results}

Patients (Table 1)

The study population included 26 patients. Their characteristics are described in Table 1. All but three received prior treatment with doxorubicin and/or ifosfamide in the adjuvant and/or metastatic setting. All the patients had documented progressive disease at initiation of treatment.

\section{Treatment}

In 7 cases, the starting dose of treatment differed from the fixed dose of $100 \mathrm{mg} /$ day orally commonly used in the study period: 5 patients received a starting daily dose of $75 \mathrm{mg}$ of etoposide, 2 patients a starting daily dose of 
Table 1 Patients characteristics and patterns of treatment $(n=26)$

\begin{tabular}{|c|c|}
\hline Characteristic & No. of patients \\
\hline Median age (years) & 49 \\
\hline Range & $23-78$ \\
\hline \multicolumn{2}{|l|}{ Sex } \\
\hline Male & 14 \\
\hline Female & 12 \\
\hline \multicolumn{2}{|l|}{ Performance status (ECOG) } \\
\hline 1 & 21 \\
\hline 2 & 5 \\
\hline \multicolumn{2}{|l|}{ Metastatic disease } \\
\hline Synchronous & 6 \\
\hline Metachronous & 20 \\
\hline \multicolumn{2}{|l|}{ Sites of metastasis } \\
\hline Lung & 20 \\
\hline Liver & 6 \\
\hline Bone & 2 \\
\hline Brain & 1 \\
\hline Nodes/skin/soft tissue & 9 \\
\hline \multicolumn{2}{|l|}{ Histopathological subtype } \\
\hline Malignant fibrous histiocytoma & 3 \\
\hline Fibrosarcoma & 3 \\
\hline Liposarcoma & 4 \\
\hline Leiomyosarcoma & 3 \\
\hline Rhabdomyosarcoma & 3 \\
\hline Synovial sarcoma & 5 \\
\hline MPNST & 1 \\
\hline Unclassified sarcoma & 3 \\
\hline Myxoid chondrosarcoma & 1 \\
\hline \multicolumn{2}{|l|}{ FNCLCC grade } \\
\hline Grade 1 & 2 \\
\hline Grade 2 & 10 \\
\hline Grade 3 & 14 \\
\hline \multicolumn{2}{|l|}{ Prior lines of chemotherapy } \\
\hline 0 & 3 \\
\hline 1 & 14 \\
\hline 2 & 7 \\
\hline 3 & 1 \\
\hline 4 & 1 \\
\hline
\end{tabular}

$150 \mathrm{mg}$ of etoposide. The median duration of treatment was 21 weeks (range 2 to 83 ).

\section{Safety}

The overall safety profile was favorable, two patients experienced grade 4 febrile neutropenia. One of them died of severe sepsis 2 weeks after starting etoposide at the daily dose of $100 \mathrm{mg}$. Eleven patients had grade 3 neutropenia, without any fever. Grade 3 thrombocytopenia was observed in two patients. Grade 3 non-hematological toxicity occurred in three patients (mucositis). A dose reduction, based on hematological toxicity, was applied in four patients who were initially treated with a starting dose of $100 \mathrm{mg}(n=3)$ or $75 \mathrm{mg}(n=1)$ of etoposide.

Efficacy (Table 2; Fig. 1)

The median follow-up was 10 months (range 0.5-57). One patient (4\%) had partial response and 11 patients (42\%) had stable disease for more than 24 weeks. The clinical characteristics of patients who experienced clinical benefit (partial response or stable disease $\geq 24$ weeks) are described in Table 2. The median PFS was 4.9 months [95\% CI: $2.9 ; 7]$. The 6 -month and the 1 -year PFS rates were $42 \%$ [95\% CI: 23; 61] and 23\% [95\% CI: 7; 39], respectively. At the time of analysis, 25 patients had died and 1 was still alive. Twenty-four deaths were the result of sarcoma and one death was related to the treatment (severe sepsis) and 2 deaths were the result of other causes. The median OS was 9.8 months [95\% CI: 6.4; 13.2]. The 6-month and the 1 year OS rates were $69 \%$ [95\% CI: 51; 87] and 31\% [95\% CI: 13; 49], respectively. Although advanced age, grade 3 and liver metastasis were associated with a trend for poor outcome in terms of PFS and OS, none of these factors reached statistical significance on univariate analysis (data not shown).

\section{Discussion}

Etoposide is supposed to have only a limited activity in STS when delivered in the context of a "maximum-tolerated dose" chemotherapy regimen [13, 14]. In this study, etoposide was not used as conventional cytotoxic drug but as anti-angiogenic agent. Indeed, preclinical studies of metronomic chemotherapy in cytotoxic-resistant tumor models have shown that tumor cell apoptosis is preceded by increased death of tumor-associated endothelial cells, indicating that endothelial cells are a primary target of metronomic chemotherapy [15]. The exact mechanisms by which metronomic chemotherapy targets selectively endothelial cells are not yet fully understood. Several studies have shown that activated endothelial cells of newly formed blood vessels are highly and selectively sensitive to very low doses of various cytotoxic drugs such as etoposide, cyclophosphamide, methotrexate, paclitaxel, vinblastine, taxanes, doxorubicin, and 5-fluorouracil [16, 17]. Interestingly, recent data have suggested that this antiangiogenic effect of metronomic chemotherapy may be also mediated by the induction of the expression of the endogenous inhibitor of angiogenesis thrombospondin (TSP)-1 [18]. 
Table 2 Characteristics of patients with clinical benefit from metronomic chemotherapy $(n=12)$

\begin{tabular}{|c|c|c|}
\hline Characteristic & No. of patients & $\%$ \\
\hline Median age (years) & 57 & \\
\hline Range & $27-76$ & \\
\hline \multicolumn{3}{|l|}{ Sex } \\
\hline Male & 6 & 50 \\
\hline Female & 6 & 50 \\
\hline \multicolumn{3}{|l|}{ Performance status (ECOG) } \\
\hline 1 & 11 & 92 \\
\hline 2 & 1 & 8 \\
\hline \multicolumn{3}{|l|}{ Metastatic disease } \\
\hline Synchronous & 2 & 17 \\
\hline Metachronous & 10 & 83 \\
\hline \multicolumn{3}{|l|}{ Sites of metastasis } \\
\hline Lung & 8 & 67 \\
\hline Liver & 3 & 25 \\
\hline Skin/soft tissue/nodes & 5 & 51 \\
\hline \multicolumn{3}{|l|}{ Histopathological subtype } \\
\hline Malignant fibrous histiocytoma & 1 & \\
\hline Fibrosarcoma & 1 & \\
\hline Liposarcoma & 2 & 17 \\
\hline Leiomyosarcoma & 1 & 8 \\
\hline Rhabdomyosarcoma & 2 & 17 \\
\hline Synovial sarcoma & 3 & 25 \\
\hline MPNST & 1 & \\
\hline Myxoid chondrosarcoma & 1 & \\
\hline \multicolumn{3}{|l|}{ FNCLCC grade } \\
\hline Grade 1 & 1 & 8 \\
\hline Grade 2 & 5 & 42 \\
\hline Grade 3 & 6 & 50 \\
\hline \multicolumn{3}{|l|}{ Prior lines of chemotherapy } \\
\hline 0 & 1 & 8 \\
\hline 1 & 6 & 50 \\
\hline 2 & 4 & 34 \\
\hline 4 & 1 & 8 \\
\hline \multicolumn{3}{|l|}{ Metronomic regimen } \\
\hline Etoposide $100 \mathrm{mg} /$ day & 8 & 67 \\
\hline Etoposide 75 mg/day & 3 & 25 \\
\hline Etoposide $150 \mathrm{mg} /$ day & 1 & 8 \\
\hline
\end{tabular}

The objective of this study was to explore whether metronomic chemotherapy, the activity of which has been already observed in other solid tumor types [19-27], warrants further investigation in STS. There is accumulating evidence that antitumor activity of angiogenesis inhibitors is better reflected in terms of parameters of disease stabilization rather than by objective response. Therefore, the endpoint of progression-free survival was considered as a valuable alternative by the EORTC group to estimate the clinical activity of this type of agents. For patients with advanced STS who have failed for first-line therapy with anthracyclines and/or ifosfamide, agents that yielded a 6-month PFS rate greater than $20 \%$ deserve additional investigation [28]. Despite their retrospective nature, our results showing a 6-month PFS rate of $42 \%$ represent the first confirmation in adult STS of the preclinical data indicating a potential activity of metronomic chemotherapy. These results reproduce also those obtained in the pediatric setting. Indeed, a recent study evaluating the safety and efficacy of metronomic etoposide in children with advanced sarcoma has reported a clinical benefit rate of $43 \%$, quite similar to that observed in our study [29].

By showing a high clinical benefit rate in a heavily pretreated population who has failed the two most active agents in STS (doxorubin and ifosfamide), our data suggest the clinical relevance of targeting angiogenesis in STS. Three recent phase II trials including patients with advanced STS have reported the efficacy profile of multikinase angiogenesis inhibitors namely sorafenib, sunitinib, and pazopanib that targets, among other receptors, VEGF receptors and PDGF receptors and which represent an alternative way to inhibit angiogenesis in STS [30-32]. As observed with metronomic chemotherapy, these agents yield low objective response rates but showed a similar relevant antitumor activity in terms of PFS.

In our series, the safety profile of metronomic chemotherapy was acceptable. Thirteen patients have experienced at least one episode of grade 3 or 4 neutropenia during the course of the treatment. These data confirm that blood cell count should be closely monitored during metronomic chemotherapy. Indeed, release of endothelial progenitor cells (CEP) from the bone marrow into the circulation in response to cytotoxic agents has been largely documented [33]. Recent data have demonstrated that maximum-tolerated dose cyclophosphamide mobilizes viable CEPs, whereas metronomic cyclophosphamide did not mobilize CEPs and, moreover, increased the frequency of apoptotic CEP [34]. These results suggest that in addition to antiangiogenic mechanisms related to the direct cytotoxicity on endothelial cells of metronomic chemotherapy, an antivasculogenic process may also be involved that is mediated through effects on reducing CEP mobilization and viability. Therefore, the metronomic dose of chemotherapy should be adapted for each patient in order to deliver the highest dose that is not associated with clinical bone marrow perturbation. Further studies are needed in order to explore the role of new circulating endothelial cells assays to monitor metronomic chemotherapy in STS patients.

Altogether, our study indicates that metronomic chemotherapy has a good clinical index profile, with minimal toxicity and significant potential benefit and, thus, warrants 
Fig. 1 Kaplan-Meier curves of progression-free survival (a) and overall survival (b) for the overall population

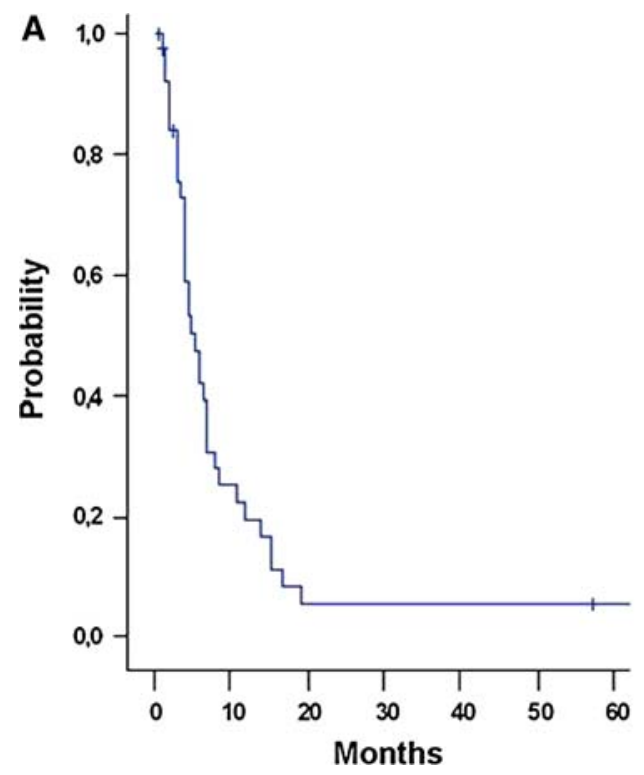

further prospective investigation in STS. These prospective studies will have to answer several questions. Indeed, our data do not allow us to identify a subgroup of patients who are more likely to benefit from metronomic chemotherapy. The level of circulating endothelial cells and the serum VEGF were suggested to be relevant biomarkers for predicting the clinical response in patients undergoing metronomic chemotherapy in breast cancer patients [22, 23] and should therefore be explored in STS patients. Moreover, whether the efficacy of metronomic chemotherapy could be increased by combining it with other antiangiogenic agents is a matter of interest. In a phase II clinical trial including ovarian cancer patients, co-administration of metronomic cyclophosphamide, and bevacizumab resulted in superior antitumor activity when compared with historic controls [26]. Promising results have also been recently reported in patients with neuroendocrine tumors treated with metronomic cyclophosphamide in combination with sorafenib [27]. Finally, the favorable safety profile of metronomic chemotherapy should lead to consider its role as a maintenance treatment after a conventional front-line therapy. Indeed, metronomic chemotherapy and conventional "maximum-tolerated dose" chemotherapy are not mutually exclusive and future studies have to determine the optimal way to use these two different approaches to improve survival of patients with advanced STS.

\section{References}

1. Blay JY, van Glabbeke M, Verweij J et al (2003) Advanced softtissue sarcoma: a disease that is potentially curable for a subset of patients treated with chemotherapy. Eur J Cancer 39:64-69

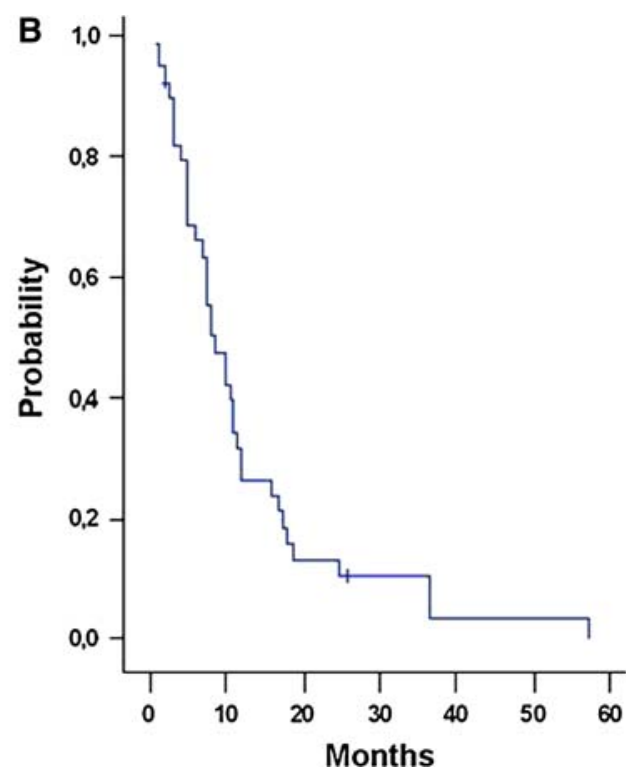

2. Brown LF, Tognazzi K, Dvorak HF et al (1996) Strong expression of kinase insert domain-containing receptor, a vascular permeability factor/vascular endothelial growth factor receptor in AIDS-associated Kaposi's sarcoma and cutaneous angiosarcoma. Am J Pathol 148:1065-1074

3. Salvin P, Lymboussaki A, Heikkila P et al (1998) Vascular endothelial growth factors VEGF-B and VEGF-C are expressed in human tumors. Am J Pathol 153:103-108

4. Onisto M, Slongo ML, Gregnanin L et al (2005) Expression and activity of vascular endothelial growth factor and metalloproteinases in alveolar and embryonal rhabdomyosarcoma cell lines. Int J Oncol 27:791-798

5. Gee MF, Tsuchida R, Eichler-Jonsson C et al (2005) Vascular endothelial growth factor acts in an autocrine manner in rhabdomyosarcoma cell lines and can be inhibited with all-trans-retinoic acid. Oncogene 24:8025-8037

6. Kerbel RS, Kamen BA (2004) The anti-angiogenic basis of metronomic chemotherapy. Nat Rev Cancer 4:423-436

7. Browder T, Butterfield CE, Kräling BM et al (2000) Antiangiogenic scheduling of chemotherapy improves efficacy against experimental drug-resistant cancer. Cancer Res 60:1878-1886

8. Rozados VR, Sánchez AM, Gervasoni SI et al (2004) Metronomic therapy with cyclophosphamide induces rat lymphoma and sarcoma regression, and is devoid of toxicity. Ann Oncol 15:1543-1550

9. Elmslie RE, Glawe P, Dow SW (2008) Metronomic therapy with cyclophosphamide and piroxicam effectively delays tumor recurrence in dogs with incompletely resected soft tissue sarcomas. J Vet Intern Med 22:1373-1379

10. Fletcher C, Unni K, Mertens F (2002) World Health Organization classification of tumours pathology and genetics of tumours of soft tissue and bone. IARC Press, Lyon

11. Trojani M, Contesso G, Coindre JM et al (1984) Soft-tissue sarcomas of adults; study of pathological prognostic variables and definition of a histopathological grading system. Int $\mathrm{J}$ Cancer 33:37-42

12. Therasse P, Arbuck SG, Eisenhauer EA et al (2000) New guidelines to evaluate the response to treatment in solid tumors. European organization for research and treatment of cancer, national cancer institute of the United States, National Cancer Institute of Canada. J Natl Cancer Inst 92:205-216 
13. Dombernowsky P, Buesa J, Pinedo HM et al (1987) VP-16 in advanced soft tissue sarcoma: a phase II study of the EORTC Soft Tissue and Bone Sarcoma Group. Eur J Cancer Clin Oncol 23:579-580

14. Bramwell VH, Mouridsen HT, Santoro A et al (1993) Cyclophosphamide versus ifosfamide: a randomized phase II trial in adult soft-tissue sarcomas. The European Organization for Research and Treatment of Cancer [EORTC], Soft Tissue and Bone Sarcoma Group. Cancer. Chemother Pharmacol 31(2):S180-S184

15. Browder T, Butterfield CE, Kraling BM et al (2000) Antiangiogenic scheduling of chemotherapy improves efficacy against experimental drug-resistant cancer. Cancer Res 60:1878-1886

16. Twardowski PW, Smith-Powell L, Carroll M et al (2008) Biologic markers of angiogenesis: circulating endothelial cells in patients with advanced malignancies treated on phase I protocol with metronomic chemotherapy and celecoxib. Cancer Invest 26:53-59

17. Bocci G, Nicolaou KC, Kerbel RS (2002) Protracted low-dose effects on human endothelial cell proliferation and survival in vitro reveal a selective antiangiogenic window for various chemotherapeutic drugs. Cancer Res 62:6938-7432

18. Bocci G, Francia G, Man S, Lawler J, Kerbel RS (2003) Thrombospondin 1, a mediator of the antiangiogenic effects of low-dose metronomic chemotherapy. Proc Natl Acad Sci U S A 100:12917-12922

19. Borne E, Desmedt E, Duhamel A, et al. (2009) Oral metronomic cyclophosphamide in elderly with metastatic melanoma. Invest New Drugs [Epub ahead of print]

20. Samuel DP, Wen PY, Kieran MW (2009) Antiangiogenic (metronomic) chemotherapy for brain tumors: current and future perspectives. Expert Opin Investig Drugs 18:973-983

21. Nelius T, Klatte T, de Riese W, et al. (2009) Clinical outcome of patients with docetaxel-resistant hormone-refractory prostate cancer treated with second-line cyclophosphamide-based metronomic chemotherapy. Med Oncol. [Epub ahead of print]

22. Mancuso P, Colleoni M, Calleri A et al (2006) Circulating endothelial- cell kinetics and viability predict survival in breast cancer patients receiving metronomic chemotherapy. Blood 108:452-459

23. Colleoni M, Orlando L, Sanna G et al (2006) Metronomic lowdose oral cyclophosphamide and methotrexate plus or minus thalidomide in metastatic breast cancer: antitumor activity and biological effects. Ann Oncol 17:232-238
24. Dellapasqua S, Bertolini F, Bagnardi V et al (2008) Metronomic cyclophosphamide and capecitabine combined with bevacizumab in advanced breast cancer. J Clin Oncol 26:4899-4905

25. Coleman M, Martin P, Ruan J et al (2008) Prednisone, etoposide, procarbazine, and cyclophosphamide (PEP-C) oral combination chemotherapy regimen for recurring/refractory lymphoma: lowdose metronomic, multidrug therapy. Cancer 112:2228-2232

26. Garcia AA, Hirte H, Fleming G et al (2008) Phase II clinical trial of bevacizumab and low-dose metronomic oral cyclophosphamide in recurrent ovarian cancer: a trial of the California, Chicago, and Princess Margaret Hospital phase II consortia. J Clin Oncol 26:76-82

27. Quintela-Fandino MA, Young A, Webster S et al (2009) Phase II trial of pharmacodynamically (PDally)-guided optimal biologic dose titration (OBDT) of sorafenib (S) in combination with metronomic cyclophosphamide (mC) in advanced neuroendocrine tumors (aNET). J Clin Oncol 27:15s (suppl; abstr 3526)

28. Van Glabbeke M, Verweij J, Judson J, Nielsen OS (2002) Progression-free rate as the principal end point for phase II trials in soft tissue sarcomas. Eur J Cancer 38:543-549

29. Kebudi R, Görgün O, Ayan I (2004) Oral etoposide for recurrent/ progressive sarcomas of childhood. Pediatr Blood Cancer 42:320-324

30. Sleijfer S, Ray-Coquard I, Papai Z et al (2009) Pazopanib, a multikinase angiogenesis inhibitor, in patients with relapsed or refractory advanced soft tissue sarcoma: a phase II study from the European organisation for research and treatment of cancer-soft tissue and bone sarcoma group (EORTC study 62043). J Clin Oncol 27:3126-3132

31. George S, Merriam P, Maki RG et al (2009) Multicenter phase II trial of sunitinib in the treatment of nongastrointestinal stromal tumor sarcomas. J Clin Oncol 27:3154-3160

32. Maki RG, D'Adamo DR, Keohan ML et al (2009) Phase II study of sorafenib in patients with metastatic or recurrent sarcomas. J Clin Oncol 27:3133-3140

33. To LB, Haylock DN, Simmons PJ, Juttner CA (1997) The biology and clinical uses of blood stem cells. Blood 89:2233-2258

34. Bertolini F, Paul S, Mancuso P et al (2003) Maximum tolerable dose and low-dose metronomic chemotherapy have opposite effects on the mobilization and viability of circulating endothelial progenitor cells. Cancer Res 63:4342-4346 